\title{
Sauver la dignité humaine : Péguy entre le bergsonisme et le personnalisme
}

\section{To Save Human Dignity: Péguy between Bergsonism and Personalism}

NeLli SoŁonko [nelli.solonko@gmail.com]

Uniwersytet Szczeciński, Pologne

\section{RÉSUMÉ}

La vie intellectuelle française du début du $\mathrm{XX}^{\mathrm{e}}$ siècle se concentre autour du philosophe Henri Bergson, enseignant à l'École Normale Supérieure et au Collège de France. Sa pensée ouvre la voie à la métaphysique en sopposant à «l'historicisme sorbonnier », étant aussi un antidote au positivisme pseudo-scientifique, au scepticisme et au relativisme scientifique. Charles Péguy, philosophe et poète français, reste un grand défenseur des cours, des écrits et de la pensée bergsoniens. Certainement, Bergson introduit Péguy à une certaine sensibilité métaphysique et à un goût de la spiritualité. La pensée philosophique et poétique de Péguy contient des germes du personnalisme. Péguy préfigure une nouvelle manière de penser, mais c'est seulement après sa mort que Jacques Maritain conceptualise la notion de personne. Emmanuel Mounier, dont le premier livre est consacré à Charles Péguy, devient le fondateur d’un courant philosophique français, le personnalisme. Dans notre article, nous recherchons la valeur de la dignité humaine dans le poème de Péguy intitulé Ève.

\section{MotS-CLÉS}

Péguy; bergsonisme ; personnalisme ; dignité humaine

\begin{abstract}
The life of French intellectuals of the early $20^{\text {th }}$ century was centered around Henri Bergson, a philosopher and École Normale Supérieure and Collège de France lecturer. Bergson's thought leads the way towards the metaphysical path and resisted 'the Sorbonnian historicism'; was an antidote to positivism, skepticism and scientific relativism. Charles Péguy, a thinker and poet, fiercely defended Bergson's lectures, writings and reflections. Certainly, Bergson must have awakened particular spiritual sensitivity in Péguy. Péguy's philosophical and poetic thought is situated between bergsonism and personalism. The elements of personalism and sensitivity to the value of human dignity can be observed in his poems and poetry. Péguy prefigured the new way of thinking. It was just after the poet's death when Jacques Maritain defined the notion of a person. Emmanuel Mounier, whose first book was devoted to Charles Péguy, became the founder of a philosophical movement, personalism. In this article, we are looking for the value of human dignity in Péguy's poem entitled Eve.
\end{abstract}

\section{KEYWORDS}

Péguy; bergsonism; personalism; human dignity

REÇU 2019-11-19; ACCEPTE 2020-01-29 
Charles Péguy (1873-1914), philosophe et poète français, fondateur et directeur de la revue $\mathrm{Ca}$ hiers de la Quinzaine partage avec ses contemporains l'enthousiasme pour la philosophie bergsonienne. À son tour, il marque un autre philosophe, le personnaliste Emmanuel Mounier.

Cet article met en relief les visions philosophiques qui fascinent, qui intéressent ou, du moins, qui marquent Charles Péguy. Ces grandes figures entre lesquelles sa pensée sétale sont, d’un côté, Henri Bergson, et de l'autre, Emmanuel Mounier, philosophe personnaliste. Bergson influence sa pensée et son imagination. Mounier découvrira chez Péguy une sensibilité envers la personne humaine. Péguy serait probablement une illustration de ce que sont le bergsonisme et le personnalisme littéraire. Nous chercherons à saisir à travers ses convictions philosophiques et dans son poème Ève la valeur de la dignité humaine.

\section{Sources d'une spiritualité}

Péguy tombe sous le charme de la philosophie bergsonienne qui a une grande influence sur la pensée, pour ne pas dire sur la vie, des intellectuels et des écrivains du début du XXe siècle. Dans une lettre du 2 mars 1914, il avoue à Henri Bergson : "Que les batailles qui se livrent autour de votre philosophie soient à ce point furieuses, cela n’a rien d'étonnant, mais qu'elles soient aussi complètement livrées à l'envers, voilà ce qui est inouï. C’est vous qui avez rouvert en ce pays les sources de la vie spirituelle. Cela ne fait aucun doute [...]» (Péguy 1970 : 48).

Bergson fascine non seulement les philosophes, mais aussi les littéraires. Romain Rolland confirme qu' « une illumination métaphysique, [...] longtemps couvée, préparée, annoncée, prit force de foi depuis 1900, aux cours de Bergson, au Collège de France » (Rolland 2015 : 36). La renommée et la critique de cette philosophie ne se propagent pas seulement en France. Bergson est invité pour des interventions dans plusieurs pays, entre autres aux États-Unis, en Grande Bretagne, Espagne, Italie, Suisse ${ }^{1}$. Dans le cercle phénoménologique de Husserl, les discussions se déroulent aussi au sujet du bergsonisme ${ }^{2}$.

Selon les propos de Raïssa Maritain, Bergson tenait ses auditeurs en suspens grâce à son éloquence et son authenticité. Mais ce n'est pas seulement son charme éducatif qui a lancé une véritable mode bergsonienne. Ses cours promettaient une vie spirituelle et intellectuelle à ceux qui ne trouvaient plus de réponse dans le positivisme scientifique, le scientisme, le matérialisme, le scepticisme ou le relativisme. Bergson fait face aux idées d'Ernest Renan et d'Hippolyte Taine. Il dépasse le déterminisme, la répétitivité et l'abstraction positivistes par les concepts de liberté, de créativité et par l'introduction des catégories métaphysiques.

Laissons témoigner Raïssa Maritain de ce grand enthousiasme que Bergson éveille dans le milieu français de l'époque : «C’est alors que la pitié de Dieu nous fit trouver Henri Bergson. Il

1 Cette mondanéité de Bergson est mise en relief dans l'article « Bergson, précurseur des mobilités académiques contemporaines?» (Dervin 2010).

2 En 1916, R. Ingarden soutient chez Husserl une thèse intitulée Intuition et intellect chez Henri Bergson. Il échange également une correspondance avec Édith Stein où la question de la philosophie de Bergson est soulevée. Dans la $70^{\mathrm{e}}$ lettre d'Édith Stein à Ingarden, on se réfère à une opinion transmise dans une revue catholique selon laquelle Henri Bergson a été à la base du nouveau courant philosophique qui rejette les découvertes spirituelles des temps anciens. Le fondement de cette nouvelle philosophie est l'intuition, qui compile l'intellect, le raisonnement et une capacité nouvelle de pressentiment. 
enseignait au Collège de France, dont les bâtiments font face à ceux de la Sorbonne. [...] Celui qui nous fit traverser la rue, celui qui nous a fait passer d'une maison à l'autre, fut justement l'ennemi déclaré de 'l'historicisme sorbonnien' - Charles Péguy. Un jour donc, ayant vu que notre déception était complète, il nous emmena au cours de Bergson. [...] Nous trouvâmes le philosophe dans l'éclat de sa jeune gloire. » (Maritain 2000 : 61-64)

Ceux qui participaient aux cours de Bergson nétaient pas guidés seulement par le snobisme ; les auditeurs y recherchaient de la métaphysique qu'ils ne pouvaient pas trouver dans le positivisme qui dominait la science de cette époque. Parmi les auditeurs, Raïssa Maritain cite en effet Charles Péguy, George Sorel, Ernest Psichari, Henri Focillon, Jean Marx, Masson-Oursel ou la poétesse Anna de Noailles.

Sa pensée, et notamment son idée du temps vécu intérieurement et subjectivement, laisse des traces dans l'écriture de Marcel Proust dans À la recherche du temps perdu (1913-1927), chez Romain Rolland dans Vie de Beethoven (1903) ou chez Joseph Malègue dans Augustin ou le Maître est là (1933).

Les athées déclarés comme Péguy, empêtrés également dans les méandres du socialisme, Jacques et Raïssa Maritain, retrouvent grâce à Bergson la foi qui pourtant dépasse la proposition de son système philosophique. Bergson influence également les penseurs catholiques comme Étienne Gilson et Jacques Chevalier.

\section{Fidélité de Péguy}

Péguy connaîtra Bergson depuis sa formation à l'École Normale Supérieure. Bientôt il lira les ouvrages majeurs de son maître Essai sur les données immédiates de la conscience (1889), Matière et Mémoires (1896) qu’il offrira « comme cadeau de fiançailles» (Azouvi 2018:43) à sa future femme Charlotte Baudouin. Il sera également son auditeur assidu au Collège de France. Il échangera une correspondance régulière avec son maître jusqu’à la fin de sa vie ${ }^{3}$. Il sera son ami dévoué et fidèle lors de son épanouissement intellectuel, il le restera au moment des attaques des thomistes, conduites par Jacques Maritain et Julien Benda contre Bergson, lors de sa mise à l'index par le décret du Saint Office 4 et lors de la compagne antisémite de l’Action française. Il semble que son dévouement ne soit pas dû seulement à des liens d'amitié. Péguy est profondément convaincu de la justesse et de la grandeur des idées philosophiques de Bergson. Il en donne le témoignage à plusieurs reprises, entre autres, dans des textes tels que Les récentes ouvres de Zola (Péguy 1987 : 243-263), Zangwill (Péguy 1987 : 243-263), mais surtout dans ceux dédiés à sa défense : Note sur M. Bergson et la philosophie bergsonienne (Péguy 1992 : 1246-1277), Note conjointe sur M. Descartes et la philosophie cartésienne (Péguy 1992 : 1278-1477). Péguy prend parti dans le débat qui se déroule autour de la philosophie de Bergson et qui repose, selon lui, sur un accueil injuste des œuvres du

3 E. Mounier évoque une profonde influence de Bergson sur Péguy: "Mais aussi il avait rencontré chez Bergson, à peine sétaient creusés pour lui les problèmes techniques, les solutions qui l'assurèrent pour toute la vie." (Mounier 2015 : 44).

4 «Trois livres de Bergson (Essai sur les données immédiates de la conscience, Matière et Mémoire, L'Évolution créatrice) sont mis à l'index en juin 1914. Pour de nombreux intellectuels et théologiens catholiques, pragmatisme, mobilisme et anti-intellectualisme caractérisent la philosophie bergsonienne, qui apparaît incompatible avec une compréhension chrétienne de la vérité de la création. » (Mounier 2015 : 69). 
philosophe. Un texte à la défense de Bergson paraît dans le Huitième cahier de la quinzième série, le 26 avril 1914, peu avant la disparition soudaine de Charles Péguy au champ d'honneur.

Si Péguy est aussi favorable à Bergson, Bergson reste d'abord indifférent et distant quant à sa production littéraire. Dans une lettre adressée à Bergson, Péguy se lamente : « vous navez jamais soupçonné le mal que vous m’avez fait le jour où vous mavez dit que vous n’aviez plus le temps de me lire » (Péguy $1970: 46)^{5}$. Bergson se rachète après la mort de Péguy et lui rend hommage à son tour : «Grande et admirable figure ! Elle avait été taillée dans l'étoffe dont Dieu se sert pour faire les héros et les saints. » (Bergson : 1970). Sans doute, la pensée de Bergson influence-t-elle considérablement la création littéraire et philosophique de Charles Péguy. Aussi Bergson est-il influencé par Péguy. Péguy introduit Bergson, le comprend et essaie de l'expliquer à son époque ${ }^{6}$. Bergson reconnaît la dette qu'il avait à l'égard de Péguy : "il a connu ma pensée la plus secrète, telle que je ne l'ai pas exprimée, telle que j’aurais voulu l'exprimer. $»^{7}$ (Bergson d'après Chantre 2000).

\section{Le bergsonisme défendu par Péguy}

Péguy se pose la question de savoir ce qu'il y a dans la philosophie bergsonienne qui puisse attirer autant de personnes. Il semble que l'attraction du bergsonisme repose sur la spiritualité nouvellement transmise. Bergson ouvre la voie aux intellectuels et aux littéraires vers une spiritualité qui n'est pas définie et enfermée dans une doctrine. Cette nouveauté de Bergson, cette manière de voir le monde autrement, viennent d'une intéressante conceptualisation de l'intuition et de la perception du temps. L'intuition sera une illumination de la conscience, une saisie de ce qui est ineffable et singulier. Elle résulte du travail intellectuel, mais elle échappe à la pensée rationnelle. Bergson l'approche à travers sa vision de la durée qui correspond au présent vécu subjectivement : «Sans doute l'intuition comporte bien des degrés d'intensité [...], mais l'esprit qu’on aura ramené à la durée réelle vivra déjà de la vie intuitive et sa connaissance des choses sera déjà philosophie ${ }^{8}$ (Bergson 1934 : 160).

Bergson envisage donc deux temporalités : l'une mesurable, abstraite, scientifique et l'autre de qualité, de conscience, d'introspection. Il tient à démontrer que le temps qui se laisse mesurer ne correspond pas nécessairement à une durée particulière. Cette dernière représente le temps vécu, le temps de l'existence qui entraîne une participation active de la mémoire. La durée a une valeur créatrice.

Analogiquement, il y a deux réalités. Celle qui se laisse décrire avec une certaine distance, par le biais de la connaissance scientifique, et celle qui est vécue intérieurement, à laquelle il est possible de parvenir seulement à travers l'intuition. Dans ce contexte, l'analyse intellectuelle reste extérieure, vu que la connaissance rationnelle se réduit seulement à une manière d'envisager les choses. Il y a donc des souches du monde qui peuvent être pénétrées seulement par la mystique et l'intuition, et non par la science et la connaissance.

$5 \quad$ Lettre du 27 février 1914.

6 Voir (Chantre 2000) ; (Mounier 1943, d'après Kisukidi 2015 : 17).

7 Ce propos de Bergson a été adressé à Geneviève Favre, la mère de Jacques Maritain, en 1915.

8 Cette réflexion vient de la conférence sur L'intuition philosophique qui a été prononcée au Congrès de philosophie de Bologne le 10 avril 1911. 
Péguy est à juste titre ébloui par l'idée du temps et de la durée caractéristique de Bergson : « [...] la révolution bergsonienne [...] a retrouvé le présent. Partout elle a restitué, réintégré la présence du présent. Partout elle a restitué le point de présence du présent. » (Péguy 1992 : 1442).

En effet, l'orientation de l'esprit vers l'instant qui dure, devient alors une vraie libération. L'esprit n’est pas perturbé par le passé qui ne lui appartient pas ou le futur qui ne se laisse pas prévoir. La durée admet une corrélation entre passé, futur et présent. Les barrières qui les séparent tombent ; une nouvelle conscience resurgit alors. Cette durée si séduisante pour Péguy lui ouvre la voie à l'intériorité et au vécu du temps qui est autre que le temps historique, linéaire et mesurable : "C’est cette profonde et capitale idée bergsonienne que le présent, le passé, le futur ne sont pas du temps seulement mais de l'être même. " (Péguy 1992 : 1254).

Si la quête spirituelle de Péguy a commencé avec Bergson, elle s’est radicalisée dans la voie chrétienne qu'il doit en quelque sorte à ce philosophe ${ }^{9}$. Nous osons dire que Péguy, aussi enthousiaste qu'il soit devant la philosophie bergsonienne, la réadapte à sa manière : « [...] dans cette position du présent, $M$. Bergson en cela même et déjà par cela seul nous réintroduit dans une situation et dans une position chrétienne. » (Péguy 1992 : 1444).

Cette leçon sur le dépassement du passé et du futur, propre aux philosophes de l'Antiquité, trouve aussi ses articulations dans le christianisme et notamment chez les mystiques qui dans le présent trouvent les traces de l'éternité. Bergson ne puise cependant pas dans la tradition, mais certaines de ses idées résonnaient avec la réflexion chrétienne sur le présent ${ }^{10}$. Péguy reste particulièrement sensible à ces connotations chrétiennes.

La réflexion bergsonienne sur le temps l'amène à méditer sur l'incarnation et sur la fragilité de l'Homme. Le système bergsonien s'attache fortement à l'idée de vie. L'élan vital, une pulsion de vie, une évolution créatrice ; c'est une force, qui prend sa source en Dieu. Toute la création de Bergson est dans le devenir ; de même la pensée appelle à une évolution, à une confrontation à la nouveauté. Dans la perception de Péguy, elle résiste à des habitudes, à une paresse de pensée (Péguy 1992 : 1253). Le tout faisant exprime l'idée capable d'agir, de créer, de concevoir. L'idée toute faite est cependant schématique, inopérante, elle laisse le monde tel quel, sans l'avoir changé. Péguy rajoute, toujours pour défendre Bergson : "Le bergsonisme a entrepris de refouler toute l'habitude comme telle, toute l'habitude organique et mentale.» (Péguy 1992: 1272). Il spiritualise même ce choix par un renvoi à des notions empruntées à l'éthique chrétienne : "Ce qu'il y a de plus contrarié au salut même, ce n’est pas le péché, c’est l'habitude. » (Péguy 1992 : 1273).

Péguy défend Bergson contre un manque de rigueur méthodologique de la pensée. Il juxtapose à la figure de Bergson celle de Descartes, qu'il juge moins cartésien dans l'ensemble de ses discours philosophiques par rapport à Bergson qui reste, dans sa perception, plus bergsonien dans ses œuvres et donc plus cohérent dans son discours : " La philosophie de M. Bergson est presque aussi mal comprise par ses adversaires que par les partisans. " (Péguy 1992 : 1246). Cette confrontation de Bergson et Descartes n'a pas pour but la dévalorisation de ce dernier. Il s'agit tout simplement de sauver Bergson du reproche d'un désordre méthodologique et, probablement, de le mettre sur le même rang des grands philosophes français : " Comme la

9 Au sujet de la durée et du christianisme voir aussi le livre Charles Péguy i Biblia. Lektura symboliczna życia i twórczości Péguy'ego (Pereira 2005) et Filozofia Henriego Bergsona w poezji Charlesa Péguy'ego (Sołonko 2019).

10 Il y a des études qui montrent que la philosophie de Bergson mène à des vérités propres au catholicisme. Henri Bergson et le catholicisme (Sertillages 1941) désigne les similitudes entre catholicisme et bergsonisme. 
philosophie bergsonienne a commencé par être une dénonciation du tout fait, ainsi la philosophie cartésienne a commencé par une dénonciation du désordre. La philosophie cartésienne a été essentiellement une philosophie de l'ordre comme la philosophie bergsonienne est essentiellement une philosophie de la réalité. » (Péguy 1992 : 1257).

\section{La morale ouverte}

L'argument fort de Péguy en faveur de la philosophie bergsonienne réside dans l'habileté de sa pensée qui correspond à la souplesse de la morale : "Ce sont les morales souples et non pas les morales raides qui exercent les contraintes les plus implacablement dures. Les seules qui ne s'absentent jamais. Les seules qui ne pardonnent pas. Ce sont les morales souples, les méthodes souples, les logiques souples qui exercent les astreintes impeccables. C'est pour cela que le plus honnête homme n'est pas celui qui entre dans des règles apparentes. C'est celui qui reste à sa place, travaille, souffre, se tait. » (Péguy 1992 : 1277).

La morale souple ne désigne pas dans le langage de Péguy une morale conformiste qui s'adapte à telle ou telle situation. Elle n'est pas non plus une morale libérale. Rappelons que le vocabulaire de Péguy est riche en références au péché et au salut. La morale bergsonienne est celle qui se défend contre les idées reçues, qui est à la recherche de la vérité, contre tous les schémas déjà proposés, préfabriqués, codifiés et acquis. C’est une morale qui réside dans l'ouverture à la créativité et dans la confrontation à une vie constamment renouvelée. Cette morale bergsonienne défendue par Péguy repose sur la liberté. Dans sa Note sur Bergson Péguy insiste sur la liberté : « La liberté, dont on dit quelle est le premier des biens, ne s'obtient généralement que par une opération de désentrave. Pourquoi la réalité, qui est peut-être un bien plus profond, ne s'obtiendrait-elle pas aussi par une opération de désentrave. Et pourquoi une opération de désentrave ne serait-elle pas une opération d'une extrême importance. » (Péguy 1992 : 1254).

L'interprétation de Bergson entreprise par Péguy témoigne aussi d'une sensibilité propre face à la question de la morale. Soit Péguy s'attendait à ce que Bergson se confronte au défi de la morale, soit sa perception de la philosophie était intrinsèquement liée aux problèmes sociaux-moraux. Cela n'empêche que Péguy rend certainement l'œuvre de Bergson plus morale qu'elle ne l'était à l'époque. N. Yala Kisukidi confirme que les idées de deux textes de Péguy : Note sur M. Bergson et la philosophie bergsonienne (Péguy 1992 : 1246-1277) et Note conjointe sur M. Descartes et la philosophie cartésienne (Péguy 1992 : 1278-1477) anticipent en quelque sorte le contenu de l'ouvrage majeur de Bergson sur la morale. Toujours selon elle, Péguy rend Bergson spirituel et il inscrit sa pensée dans l'ordre du péché et du salut (Kisukidi 2015 : 16). Ses idées évolueront vers le christianisme. Or, Bergson entamera ultérieurement la question de la morale, et ce après la mort de Péguy.

Les premières et les plus importantes œuvres de Bergson n’abordent nullement les problèmes éthiques, mais le climat social et philosophique du début du $\mathrm{XX}^{\mathrm{e}}$ siècle exigeait en quelque sorte une prise de position. Ses disciples attendaient depuis longtemps un livre sur la morale. Confronté à ses adversaires qui lui reprochent l'absence de la question morale (Prelorentzos 2007 : 108-109), Bergson lève enfin le défi. Dans sa correspondance il s'aperçoit de l'accroissement de l'intérêt pour les sujets « sociaux, moraux et religieux » (Bergson 2002, d'après Prelorentzos 2007 : 109). 
L'ouvrage de Bergson Les deux sources de la morale et de la religion paraît en 1932, bien après la mort de Charles Péguy. Bergson y développe l'idée de religions dynamiques et statiques, qui se différencient selon leur source. Il procède également à une distinction entre la morale ouverte et de la morale close. Elles évoquent, d'une part, la pression et l'influence de la société, qui proviennent de l'instinct et de l'intellect, et, d'autre part, l'amour de tous, qui provient de l'intuition et de la force créative. La morale close propre aux âmes closes consiste à répéter le devoir et ce qui est prescrit comme bon ; tandis que la morale ouverte des âmes ouvertes procède de la création et de la charité, ne se concentrant pas sur l'individu-même, mais agissant pour le bien des autres. Il n’est donc pas étonnant que selon Bergson « la morale de l'Évangile est essentiellement ouverte» (Prelorentzos 2007 : 126). La moralité et la religion ouvertes dépassent la nature et marquent les mystiques qui ont éprouvé ou expérimenté Dieu (Gadacz 2009 : 142). Bergson aborde le sujet de la morale et de l'obligation pour montrer que c'est la foi mystique qui est une source de créativité. La morale ouverte caractérise les héros et les saints. Charles Péguy " a rejoint, aux yeux de Bergson, les héros et les saints dont il est question dans Les Sources " (Soulez, Worms 2002 d'après Prelorentzos 2007 : 162).

Nous admettons que certaines idées bergsoniennes, malgré leur évolution incontestable, ont pu resurgir du vivant de Péguy. La morale souple ou ouverte a toujours fasciné Péguy qui a vu dans Jeanne d'Arc ${ }^{11}$ - qui n'agit pas pour son propre bien, mais pour le bien des autres - la grande héroïne de ses poèmes. Les valeurs défendues par Péguy résultent fortement de cette confrontation à la philosophie bergsonienne. L'élan vital, le tout faisant, la durée, l'introduisent à la formation de ces propres valeurs, concentrées autour de la vie humaine et de l'espérance de la vie éternelle. Cette idée est renforcée par la conviction de Nadia Yala Kisukidi : " Péguy fit de la philosophie bergsonienne de la liberté et de la vie créatrice une philosophie de l'espérance - cette philosophie devant se déployer plus tard, solide et désormais consciente d’elle-même, dans Les deux sources. » (Kisukidi 2015 : 16)

\section{Le personnalisme précaire}

Jacques Maritain, initialement fasciné par la philosophie de Bergson ${ }^{12}$, l'abandonne finalement : elle n'est pas conforme à la vérité de la foi catholique. Maritain « a pris conscience de l'incompatibilité de certaines thèses bergsoniennes avec la valeur de vérité des énoncés de la foi » (Cottier : 312). Il s'acharne sur l'intuition bergsonienne et sa critique de l'intelligence, il rejette son idée de liberté qu'il trouve plutôt spontanée et irréfléchie. Les actes libres qui déterminent une personne ont pour base une maîtrise de la volonté (Maritain 2000 : 66). Maritain se dirige vers la philosophie de Saint Thomas et ceci à l'encontre de Bergson. À la même époque, le Pape Pie X critique la doctrine moderniste dans son encyclique Pascendi Dominici Gregis (1907). En passant, sans

11 «Bergson reconnut à son tour, au moment de la rédaction des Deux sources de la morale et de la religion, la dette qu'il avait à l'égard de Péguy : le couplage 'mécanique et mystique' qui clôt le livre du premier devait beaucoup à l'opposition dynamique, chez le second, de la 'mystique' à la 'politique' » (Chantre 2000).

12 Il faudrait tout de même remarquer qu'aussi bien Étienne Gilson que Jacques Maritain ont été des étudiants assidus de Bergson. Mais c'est au cours de leurs recherches scientifiques ultérieures que tous les deux se sont convertis au thomisme. 
mentionner explicitement Bergson, il désapprouve certaines de ses idées philosophiques. En effet, la philosophie bergsonienne ne répond pas dans sa totalité à la doctrine chrétienne. Pie X invite cependant à la lecture des écrits de Saint Thomas d'Aquin. Jacques Maritain cède à cet encouragement. Comme l'écrit le cardinal George Cottier, "la découverte de saint Thomas 'avait achevé de le guérir du bergsonisme' et lui avait manifesté sa vocation intellectuelle. Elle représente pour lui 'un nouveau printemps philosophique'» (Cottier 2007 : 316). Péguy ne prend pas ce chemin malgré la confrontation aux thèses thomistes de Maritain. Saint Thomas est pour lui un grand philosophe, mais un philosophe du passé (Péguy 1992 : 1463). Il se réserve pourtant le droit à une certaine liberté dans l'interprétation du catholicisme, d’où une incompréhension entre son ami, Jacques Maritain, philosophe thomiste, et lui. Selon Damien Le Guay, le conflit confessionnel entre les deux philosophes résulte de leurs caractères opposés. Chacun des deux envisage le catholicisme d'une manière différente (Le Guay 2001 : 342).

Incontestablement, le thomisme a apporté une réflexion importante sur la personne humaine et sa dignité. Péguy s'inscrit paradoxalement dans cette ambiance et parmi ces penseurs qui ont élevé la valeur de l'Homme. Il organise sa pensée autour de certains concepts qui sont chers aux personnalistes. Il tient absolument à la valeur de la personne, ce qui se laisse percevoir dans sa transgression du dualisme propre à la philosophie cartésienne.

Peut-être Péguy hérite-t-il du bergsonisme son attachement aux valeurs de la personne, mais il l'articule à sa propre manière, car il semble que Bergson arrive à introduire délicatement le personnalisme, entre autres, à travers maints articles sur la personnalité. Selon Camille Riquier, auteur de Bergson et le problème de la personnalité : la personne dans tous les états (Riquier 2007 : 193-214), les idées principales du philosophe comme durée, mémoire et élan vital convergent dans un être singulier qui est la personne.

La pensée autour de la personne humaine se forge peu à peu après la mort soudaine de Péguy. Les philosophes de son époque essaient de prendre leur distance avec l'individualisme bourgeois et le collectivisme et la masse (Guéna 2016 : 13) ; ainsi, s'efforcent-t-il de conceptualiser la personne humaine en l'attachant au spirituel, si cher à Péguy.

Si la philosophie de Bergson a marqué Péguy, ses écrits et sa personne en influenceront bien d'autres. Mounier, une grande figure française et le fondateur du personnalisme, voyait plus particulièrement les germes du personnalisme en la poésie de Charles Péguy. Il importe par conséquent de mettre en relation Emmanuel Mounier et Jacques Maritain, son ami, grand thomiste, qui, par le biais du thomisme, cherchait la valeur de la personne humaine, et de conduire notre réflexion dans ce sens.

Ainsi, pour Jacques Maritain : "l'homme est un individu qui se tient lui-même en main par l'intelligence et la volonté ; il n'existe pas seulement d'une façon physique, il y a en lui une existence plus riche et plus élevée, il sur-existe spirituellement en connaissance et en amour ». (Maritain 1942, d’après Guéna 2006 : 13).

Maritain, thomiste, envisage une personne autonome, dotée d'intellect, étant de nature intellectuelle et capable de dominer ses actions, et il la met en contraste avec l'individu, soumis à la matière et aux lois de la nature (J. Maritain 1925 : 28-29). L'homme devient une personne au fur et à mesure. Pour Emmanuel Mounier « une personne est un être spirituel constitué comme tel par une manière de subsistance et d'indépendance dans son être ; elle entretient cette subsistance par son adhésion à une hiérarchie de valeurs librement adoptées, assimilées et vécues par un engage- 
ment responsable et une constante conversion ; elle unifie ainsi toute son activité dans la liberté et développe par surcroît, à coup d’actes créateurs, la singularité de sa vocation » (Mounier 2000, d'après Guéna 2006 : 13-14).

Les deux philosophes, à travers l'échange de leur correspondance (Maritain, Mounier 2016), essaient de saisir le fond de la pensée de Péguy. Maritain a connu personnellement Péguy. Il l'a également lu. Mounier cherche à écrire un livre sur le poète qui par ailleurs sera son premier.

Dans son "Personnalisme", Mounier évoque Charles Péguy parmi les précurseurs du mouvement. Il le place à côté de Maritain, de Thomas d'Aquin et de Gabriel Marcel. Selon Mounier, Péguy exprime dans son lyrisme les mêmes sujets que son livre. Au centre de son ouvrage se trouve le thème "de la personne » (Mounier 1965 : 16). Pour Mounier, Péguy pense l'humain intégralement (Mounier 2015), il pense sa faible nature et son essor spirituel : " Il sait que le spirituel est premier, et il a montré qu'il le met par-dessus tout. Mais il sait aussi que dans l'ordre naturel l'esprit n'est jamais séparé d'une matière et que la connaissance naturelle veut que ce soit de l'étreinte du particulier que jaillisse la contemplation de l'universel. [...] Depuis longtemps, personne n'avait souligné avec autant de précision ni surtout chanté avec autant d'intensité poétique cet intime embrassement du ciel et de la terre » (Mounier 2015 : 148).

Le poème Ève véhicule les notions qui soutiennent l'expression de ce qui fonde la dignité humaine, sans pour autant la désigner directement.

\section{Le poème Ève : les interlocuteurs}

Ce dernier poème de Charles Péguy plonge l'esprit dans les temps symboliques et bibliques et renvoie au Livre de la Genèse : à la mère des vivants, au bonheur du paradis et à la disgrâce du péché originel. D’habitude, les grands théologiens et philosophes, lorsqu'ils se réfèrent à l'idylle paradisiaque, traitent plutôt du premier Homme, d’Adam ; ils mentionnent parfois les deux, Adam et Ève. Et si Paul de Tarse, Grégoire de Nysse, Bonaventure, Kierkegaard et Bernard de Clairvaux apportent des éclaircissements pour la compréhension d'Adam, du premier Homme et de son histoire, il est rare qu'ils évoquent la seule figure d'Evve ${ }^{13}$. Ève demeure cependant dans la conscience poétique plutôt une femme mortelle, déchue, séductrice et tentatrice ${ }^{14}$. Péguy renoue certainement avec la tradition romantique, car Victor Hugo, qu'il apprécie, revient avant lui à Ėve. Son changement concerne le mode de représentation iconique. Péguy réhabilite Ève. Il la trouve digne de la compassion de Jésus.

Ce long poème, d’environ huit mille vers, débute par une révélation des interlocuteurs :

13 Le recueil des textes intitulé La figure d'Adam rassemble une réflexion sur ces textes déjà fondateurs de l'histoire de la pensée. Parmi eux, seul celui de Camille Riquier Ève dans « Ève » - Lecture de Charles Péguy, (Riquier 2015 : 183-205) porte uniquement sur Ėve et développe une réflexion sur le poème de Charles Péguy.

14 Dans son article intitulé De l'Ève biblique à l'Ève de Péguy, Cécile Hussherr revient à de maintes représentations d'Ève dans la littérature à partir des mystères médiévaux aux prérafaélites. Hussherr évoque Christina Rosseti, Dante Gabriel Rossetti, Maurice Scève, Guillaume de Salluste du Bartas. Elle donne des exemples de cette héroïne des poèmes romantiques : Blake (The Ghost of Abel), Byron (Caïn, A Mystery), Hugo (Les Malheureux). Ève est effrayée devant de nouvelles chutes du monde. (Hussherr 2007 : 13-28). 
Jésus parle.

- O Mère ensevelie hors du premier jardin,

Vous navez plus connu ce climat de la grâce,

Et la vasque et la source et la haute terrasse,

Et le premier soleil sur le premier matin. (Péguy 1957 : 935).

Le poème Ève commence par une invocation de Jésus " $\mathrm{O}$ mère... " ; par la suite, les paroles du poème s'adressent à elle, à Ėve. Jésus, ou le poète qui se confond avec lui, l'enveloppe d'un regard du Sauveur. Désormais, son sort change. Ève n'est plus inscrite dans l'histoire de la damnation. Elle fait partie de l'histoire de l'Incarnation et du Salut. L'Ancien Testament est relu à la lumière du Nouveau. Jésus de la fin des temps rencontre celle qui est la Mère des vivants. Le poème ne renoue donc pas seulement avec le Livre de la Genèse, il fait référence à l'Apocalypse.

Et je vous aime tant ô mon âme, ô ma mère,

Première assujettie aux lois de pauvreté,

Première assujettie à la loi de misère,

Première assujettie aux lois de liberté. (Péguy 1957 : 1004).

Jésus pose un regard infiniment attristé sur cette Ève chassée du paradis. Il est près d’elle, ne néglige en rien sa situation et ne peut ignorer sa douleur. Il déplore son état et sa disgrâce. Il s'apitoie sur son sort, mais Il ne la juge pas et ne la condamne pas.

Les regrets qu'il manifeste à son égard, ne le mettent aucunement en position de juge. C’est comme si Jésus était plein de compassion devant la première femme, comme s'il comprenait son inquiétude et ses préoccupations face au désastre du péché. Jésus qui salue Ėve ne détruit pas sa création, mais il déploie devant elle une recréation, une nouvelle possibilité, le Salut. Nous osons dire qu’au contact de Jésus Ėve acquiert sa dignité ; elle devient une personne. Sans Ėve et sans son histoire, il n'y aurait pourtant point de Rédemption. La rencontre d'Ève et de Jésus devient donc une conjonction du temps et de l'éternité, de deux réalités distinctes.

Ainsi, Jésus renouvelle l'histoire, change son destin et ouvre sur l'éternel. Pour Katarzyna Pereira, la présence de Jésus, qui inaugure le poème, oriente tout le passé vers l'éternité (Pereira 2005 : 64). Grâce à sa présence, comme le remarque Riquier, l'ordre créé se heurte à l'ordre divin (Riquier 2015 : 190). Jésus vient sauver ce qui a été perdu.

\section{L'origine}

Le poète aime revenir à ces temps paradisiaques, à la beauté d'un monde nouvellement créé. La quiétude et le bonheur émanent des quatrains d'alexandrins de Péguy:

La face de la terre était alors si blonde

Que les blés déroulés faisaient de longs cheveux.

Et la beauté de l'âme et la beauté du monde

Fût descendue ainsi jusque chez nos neveux. (Péguy 1957 : 1026). 
Des pages entières contiennent une description idyllique de la béatitude édénique d’antan. Pour l'exprimer, le poète a besoin de recourir aux métaphores, comme celle de « la terre [...] blonde, les blés déroulés [...] de longs cheveux ». Et, Mounier d’argumenter de cette manière : "La création est un langage figuré que Dieu parle à l'homme dans l'univers » (Mounier 2015 : 63). Le charme du paysage reste en lien avec la grâce. «La beauté de l'âme » et «la beauté du monde » vont de pair, séclaircissent réciproquement et sont en harmonie. Le monde extérieur et intérieur restent unis.

Ce retour au bonheur, à la quiétude et à l'état de grâce du paradis, ramène le lecteur à la vitalité de l'origine et du commencement. Les animaux pleins de vie jouent joyeusement. Leur "vigueur» est « éternelle », leur « force originelle » réside dans le « calme», la « lenteur solennelle».

Et les bondissements de la biche et du daim

Nouant et dénouant leur course fraternelle

Et courant et sautant et s'arrêtant soudain

Pour mieux commémorer leur vigueur éternelle,

Et pour bien mesurer leur force originelle

Et pour poser leurs pas sur ces moelleux tapis

Et ces deux beaux coureurs sur soi-même tapis

Afin de saluer leur lenteur solennelle. (Péguy 1957 : 935).

Ce monde lointain, paradisiaque, est inscrit quelque part dans la mémoire profonde du poète qui avoue lui-même décrire le "paradis terrestre » (Péguy 1992 : 1222). La beauté du paradis évoque en effet la beauté de la terre de l'enfance de Péguy, commémorée dans son poème Présentation de la Beauce à Notre Dame de Chartres. Cette ressemblance fut d'ailleurs remarquée par Cécile Hussherr dans De l'Ėve biblique à l'Êve de Péguy (Hussherr 2007 : 13-23). Le souvenir de l'enfance demeure donc idyllique et innocent. Pour Bergson, et il se peut que pour Péguy aussi, l'enfant est encore tout près de la nature et plus près de la vie et de la réalité profonde.

Certes, l'image de l'origine reste symbolique, propre aux textes bibliques. Le poème renoue avec l'expression métaphorique du livre de la Genèse. Dans ce sens-là, Péguy n’invente rien de nouveau. Il réécrit les vérités chrétiennes. Selon le commentaire de Bruno Latour : « en lui l'origine réapparaît pour ce qu'elle est : neuve et ancienne » (Latour $2015: 360$ ). Les formules répétitives et persistantes ( "la première argile et la première terre ", " la première terre et l'argile plastique », « cette grasse argile où Dieu les modela », « cette grasse terre où Dieu les façonna ») creusent dans notre mémoire un chemin vers notre origine. Elles font penser aux récits bibliques sur la création et, plus précisément, à sa deuxième version. «Le Seigneur Dieu modela l'homme avec de la poussière prise du sol. Il insuffla dans ses narines l'haleine de vie, et l'homme devient un être vivant " $(\mathrm{Gn} 2,7)$. Par un retour à Ève, le poète accentue la dignité de l'Être humain et de la vie en général. Le Péguy bergsonien protège la vie, en tant que seule valeur qui pourtant nécessite une orientation vers l'au-delà. Les traces de l'influence bergsonienne laissent leurs empreintes non seulement dans la prose, mais aussi dans la poésie de Péguy:

Et que l'être de Dieu remonte incessamment

À son niveau de force à la même altitude [...] 
Et que l'être de Dieu recroît incessamment

À son niveau de vie à la même altitude. (Péguy 1957 : 992).

Mais chez Péguy, cette vie ne s'achève pas après la mort. Les personnalistes dépassent le déterminisme de l'existence. Mounier et Marcel oscillent autour d'une conviction que « Je suis plus que ma vie $»^{15}$ (Mounier 1965 : 85). Dans le poème Ėve, la mort marque un retour à l'origine, le retour aux mains du potier qui modèle l'homme comme l'argile pour son bien :

Heureux ceux qui sont morts, car ils sont retournés

Dans la première terre et la première argile.

Ils se sont remoulés dans le moule fragile

D’où le pouce d’un Dieu les avait démoulés. (Péguy 1957 : 1029).

Dans le fragment évoqué, le poète choisit un ton qui s'inscrit dans la poétique biblique. C’est une paraphrase du «Serment sur la Montagne ». Il est clair pourtant que la formule " Heureux ceux qui sont morts " n'est pas directement prise du texte biblique sur " les béatitudes ", et en comparaison aux tendres dénominations de l'Évangile de saint Matthieu (« heureux les pauvres», " les doux ", " qui pleurent », " qui ont faim », " les miséricordieux », " les cœurs purs », " qui font œuvre de paix ", "qui sont persécutés ») sonne douloureusement, comme un chant funèbre. Le poète introduit l'inquiétude dans ses textes si paisibles. Il distingue et sépare brutalement par cette mort le temps terrestre et le temps paradisiaque. La mort constitue le retour à l'état premier et à l'éternité.

Certaines idées bergsoniennes résonnent constamment dans la poésie de Péguy. Le monde tangible ne se laisse pas seulement décrire selon les règles de la logique et de la science. À part ce temps compté et mesuré seconde par seconde, minute par minute, il existe une autre temporalité qui est subjective et vécue. À travers ce monde tangible et naturel qui fonctionne selon une loi logique, ressurgit la promesse de l'éternité. Le temporel de Péguy ouvre la voie à une autre réalité qui ne finit pas, qui dure. Le temps conditionne même en quelque sorte l'éternité (Mounier 2015 : 147). Péguy réoriente Bergson. À son éloge de vie, il rajoute une pointe chrétienne. Ainsi, par son lyrisme le poète nous introduit à une sorte de contemplation :

Et l'éternité même est dans le temporel

Et l'arbre de la grâce est raciné profond

Et plonge dans le sol et touche jusqu'au fond

Et le temps est lui-même un temps intemporel. (Péguy 1957 : 1041).

Il y a une confrontation entre ce qui relève du divin et ce qui relève du terrestre, entre le créé et le spirituel. Cette fragilité est caractéristique des personnalistes.

15 C'est une citation qui vient de Gabriel Marcel et qui est mise en relief par Mounier dans Le personnalisme. (Mounier $1965: 85)$. 


\section{Le corps et l'esprit}

Péguy se soucie de transgresser la dualité du corps et de l'esprit, du matériel et du spirituel, du temporel et de l'éternel. Ceci est particulièrement visible dans le poème Ève. Mais cette transgression ne se produit pas automatiquement. Elle relève de l'ordre de la rédemption et de l'ordre de la grâce. Jean-Pierre Sueur attire notre attention sur l'unité de la Trinité vécue au paradis et une opposition visible dans la dualité du corps caractéristique du temps après la chute (Sueur 2007 : 85-106). Dans son commentaire d'Ève, dicté à son ami Lotte et publié sous le pseudonyme de J. Durel, Péguy confirme ce à quoi il tenait : « il se plaçait résolument en ce point central, doublement axial, par où tout passe. Il se plaçait instantanément dans l'axe du spirituel et dans l'axe du charnel, dans l'axe du temporel et dans l'axe de l'éternel. Il se donnait ensemble le maximum d'homme et pour ainsi dire le maximum de Dieu. Et Verbum factum est : c'est dire qu'il se plaçait au cœur même de l'Incarnation " (Péguy 1992: 1217-1218). Par cette attention particulière portée à Ėve, il exprime non seulement la nécessité de la référence à la spiritualité, mais aussi un grand respect de la nature : " (Jésus) est venu superposer à l'ordre de la nature l'ordre de la grâce mais non point par l'avilissement de l'ordre de la nature " (Péguy 1992 : 1228). Dans cette optique, la nature est comme élevée par le spirituel. Cet aspect spirituel renforce la dignité humaine (Granat 1985 : 562). Le poète contrebalance les deux : la spiritualité se fonde sur la vie terrestre et humaine. Cette unité du corps et de l'esprit préoccupe particulièrement les personnalistes ; ils envisagent la personne comme complète et intégrale. Rien d’étonnant donc si Emmanuel Mounier, dans son livre sur Péguy, fait ressortir cette problématique et l'accentue. Péguy l'exemplifie ainsi :

Car le surnaturel est lui-même charnel,

Et l'arbre de la grâce est raciné profond

Et plonge dans le sol et cherche jusqu'au fond,

Et l’arbre de la race est lui-même éternel. (Péguy 1957 : 1041).

\section{L'innocence et la dignité humaine}

Ce qui a été au commencement, cet état premier, avant le péché, c'est l'état d'insouciance d'Ève, c'est l'état de grâce, c'est l'état d'une pureté ou d'une innocence.

Vous n'avez plus connu l'innocence du monde

Et les greniers bondés jusque sur le portail.

Vous navez plus connu cette race féconde

Et les prés débordants d’un immense bétail. (Péguy 1957 : 944).

Il faudrait souligner le fait que Péguy névoque pas seulement le thème de l'innocence dans le poème Ėve. Il s'y penche d’abord dans Le Mystère des Saints Innocents (Péguy 1957 : 671-823) qui met en exergue l'épisode évangélique où Hérode tue les enfants incapables de commettre un moindre crime. L'innocence et la nudité sont donc des symboles récurrents dans la création poétique de Péguy. 
Le poète cherche donc justement cette pureté chez la Mère des vivants, Ève. L'innocence est l'état premier, originel de l'Homme. Dans le langage symbolique en référence au récit biblique de la création, l'innocence est en rapport avec la nudité, la simplicité et avec la liberté qui expriment une relation sans barrières au Créateur. Cet état originaire est valorisé par le poète. C’est autour de la valeur de l'innocence que se constitue Ève. L'innocence serait cet état rêvé du paradis, l'état premier des premiers parents.

Seule vous le savez, nos vertus d'aujourd'hui

Ne valent pas le quart de l'antique innocence.

Et les moralités de notre morne ennui

Ne valent pas le quart de l'antique puissance. (Péguy 1957 : 1006).

Il se pose cependant la question de savoir en quoi cet état paradisiaque est parlant ou significatif. Cet état d'innocence n'est envisageable qu'en tant quétat symbolique avant le péché originel. $\mathrm{Au}$ commencement, le regard des premiers parents n'est donc pas obscurci par le péché, signe de division et d'esclavage. Adam et Ève vivent dans un état de grâce, dans une parfaite liberté, unité et relation entre eux-mêmes et Dieu, leur créateur. La participation à la vie des Personnes Divines admet une dignité humaine singulière (Granat 1985 : 583).

Les premiers parents se trouvent dans une communication idéale. Comment donc expliquer la force de cette innocence qui prévaut sur d'autres vertus ? L'Homme au paradis vivait dans la proximité de Dieu. Cette proximité était parfaite et sans troubles. Péguy se déplace aussi vers l'ontologie de l'Être, malgré son engagement vif dans la cause historique. Ève a perdu la plénitude de la grâce, l'innocence, la liberté et le rapport direct à Dieu. La conscience de cette perte redonne à l'innocence de la valeur, accentue l'importance de la liberté. Les Pères de l'Église, qui évoquent cette réalité, se réfèrent au temps paradisiaque et symbolique pour expliquer que c'est au paradis que l'Homme a obtenu cette liberté incontestée de choisir entre le bien et le mal. Le personnalisme revient maintes fois sur la liberté qui est un attribut de l'Homme ; la liberté est intrinsèque à l'Homme : elle lui appartient. Elle contribue à sa dignité.

Pourquoi alors y revenir? Par ce retour, le poète montre et souligne la splendeur initiale de la création. L'Ėve du paradis nous rappelle que toute création est en principe bonne ${ }^{16}$. La source et l'essence de l'Homme ont une valeur incontestable. Par ce procédé du retour aux sources le poète sauve la dignité de la Mère des vivants. Cette bonté de la création et la valeur de la création en soi, ne restentelles pas toujours objet de préoccupation pour le Créateur qui protège la vie et qui aime cette vie?

Il est étonnant de voir cette innocence chez Ève. Péguy a pourtant consacré de longs vers à la Vierge Marie (Péguy 1957 : 889-924), considérée dans la théologie catholique comme sans péché originel, innocente et pure. Mounier retient dans son livre sur La Pensée de Charles Péguy une strophe de La Tapisserie de Notre Dame qui fait l'éloge de la nudité de la Vierge Marie de Chartres :

Voici la nudité, le reste est un vêtement.

Voici le vêtement, tout le reste est parure.

16 Nous tenons de Jean-Paul II la mise en valeur de l'innocence et la bonté initiale de l'Homme soulevées dans un cycle de catéchèse paru sous le titre Homme et femme Il les créa. Une spiritualité du corps, Paris, Cerf, 2004. 
Voici la pureté, tout le reste est souillure.

Voici la pauvreté, le reste est ornement. (Péguy 1957 : 903).

Mounier se penche sur quelques vers pour relever l'esthétique classicisante de pureté qui reste en lien avec le défi moral qu'il s'impose. Le poète recherche méthodiquement une sincérité. Selon le philosophe : " au plus profond cette méthode rejoignait une âme et une éthique " (Mounier $2015: 83)$.

Ce qui veut dire que la pureté - ajoutons encore l'innocence - doit se travailler à travers une ascèse. Il s'agit donc d'étudier, toujours selon Mounier, la sincérité dans son œuvre et dans l'œuvre des autres.

Péguy propose donc de réintégrer l'innocence dans l'ouverture à la Rédemption de Jésus.

\section{Les Valeurs divines}

Lorsque le poète prête sa parole à Dieu, il esquisse le Créateur tel qu'il L'imagine, tel qu'il Le conçoit. Son jeune Dieu se reflète dans sa création, surtout dans l'homme

Et Dieu lui-même jeune ensemble quéternel

Regardait ce que c'est que les jeux du jeune âge.

Calme et laissant poser son regard paternel

Il se considérait dans l'homme son image. (Péguy 1957 : 940).

Péguy glisse ainsi dans la bouche de Dieu des valeurs qui lui sont chères et qui sont inscrites également dans le judaïsme et le christianisme. Dieu a créé l'homme à son image. De cette conviction fondée sur la foi du judaïsme et du christianisme découle le principe de la dignité de l'homme ${ }^{17}$. L'homme porte en lui des traits de Celui qui l’a créé. Désormais, il est lié à son Créateur et il se distingue du reste de la nature créée, il porte en lui la valeur suprême. L'homme en soi constitue une valeur.

Comme le Dieu de Péguy est trinitaire, et Un, tel qu'il est évoqué surtout dans La Suite d'Ėve, dans les fragments, qui n’ont pas été publiés du vivant de l'auteur, il tient aussi à la personne humaine dans son intégralité.

Pour le personnalisme chrétien, tel que Mounier le définit, toutes les valeurs proviennent d'une «Personne suprême» (Mounier 1965 : 86). Le personnalisme ne défend pas telle ou telle autre valeur. Certes, il met en lumière le respect de la liberté humaine. Ce qui semble pourtant important, c'est la corrélation d'une personne et des valeurs (Mounier 1965 : 88). La personne se réfère aux valeurs qui naissent, se découvrent et s'affirment dans l'intimité personnelle.

La morale de Péguy a probablement pour but de prêter attention à l'être humain en général et à la dignité humaine en particulier. Ce but pourtant n'est pas tout à fait clair ou explicite. Il n'est pas ouvertement exprimé. Ses germes ou ses traces sont à retrouver dans son parcours poétique et philosophique.

17 Chantal Delsol prouve, entre autres, dans son livre intitulé Les Pierres d’angles. À quoi tenons-nous? (Delsol 2014 : 21), que la croyance détermine une anthropologie. Ainsi, l'homme, conçu comme personne, dérive de la foi chrétienne. 
Il est très caractéristique de Péguy d’opérer sur des symboles et ce n'est pas seulement dans le poème Êve. Il choisit justement les symboles qui lui sont propres, des symboles parlants. Ainsi, il attire l'attention sur ce qui est essentiel pour lui. Il y a chez Péguy des figures symboliques qui constituent des références stables et qui s'imprègnent au fur et à mesure du sens ; c'est ici le cas de l'enfant et de la femme. C'est sur cette faiblesse finalement que le monde se maintient et nous renvoie alors à une espérance. Le Dieu de Péguy - qui porte des traits humains - a du mal à choisir entre ce qu'Il chérit.

Il mise ainsi sur la jeunesse et la vieillesse, sur la fragilité.

Et s'il n'aime pas mieux une souple jeunesse.

Et s'il n'aime pas mieux les dures fermetés.

Et s'il n’aime pas mieux une belle vieillesse.

Et s'il n’aime pas mieux les dures pauvretés. (Péguy 1957 : 1074).

Le Dieu de Péguy hésite dans Son Amour entre la jeunesse et la vieillesse, entre la fermeté et la pauvreté. Ces états ne lui sont pas étrangers, car la vie entière lui est précieuse. Avant tout, Il aime sa création et Il aime la vie. Le poème Ėve fait retourner à la source de vie. Ėve devient mère des vivants après la chute, elle est mère hors le paradis. Hawwah signifie en hébreu "vivante " ou "source de vie ». La figure symbolique de la "première mère », « mère des vivants ", met en valeur la vie. Péguy s'attache, comme son maître Bergson, à la notion de vie. Mais c'est la misère qui attire son regard. Dans son Amour, Dieu de Péguy se penche sur l'humanité empêtrée dans le péché.

\section{La chute dans le temps}

La première femme, mère des vivants, porte le joug de l'impuissance et de la pleine conscience du mal et du péché ; et pourtant, elle a connu la valeur suprême et le bonheur des relations :

Vous regardez monter cette double impuissance,

L'impuissance d'aimer et celle de haïr.

Vous regardez monter cette double licence,

La licence d’aimer et celle de trahir. (Péguy 1957 : 973).

Péguy ne condamne pas Ėve dans son poème, mais il ne la déclare pas innocente non plus. Contre les théories théologiques actuelles qui veulent disculper Ėve de la faute, Péguy ne négocie pas sur le péché. Il envisage sa réalité et ses conséquences. Le poème Ėve traite de ce bonheur premier et de cette innocence première, mais aussi des valeurs perdues. Nous avons affaire à une tension qui se crée entre l'innocence et le péché, entre la liberté et l'esclavage, entre la béatitude et l'inquiétude. Ève, après sa chute, est donc une impuissante « aïeule de l'esclave » (Péguy 1957 : 986), tournée vers la fugacité du monde :

Vous n'avez plus connu que des bien périssables,

Et la succession et le vieillissement. 
Et la procession des mots ineffaçables.

Et le regard voilé d’un appauvrissement. (Péguy 1957 : 946)

Ève « chute dans le temps ", pour paraphraser le titre du livre d'Emil Cioran (Cioran 1964). Après avoir donc perdu cette innocence première, elle range et elle compte. Et Péguy de continuer des vers qu'il répète inlassablement, comme pour souligner la persistance de cette préoccupation après le péché : "vous avez pu compter, inlassable économe » (Péguy 1957 : 989), "vous rangez le silence et le drap funéraire " (Péguy 1957 : 987). Sous le pseudonyme de Durel, il essaie de nous convaincre qu'après cette chute dans le temps, la tâche de l'homme consiste à faire le ménage, également celui de notre âme (Péguy 1992 : 1220).

Aevum en latin signifie un siècle. La convergence du son du prénom "Ève » et d'aevum, fait que cette mère biblique renvoie à cette longue et lourde histoire des humains.

L'histoire est un sujet repensé à maintes reprises par Péguy. S’il n'a pas réalisé le projet de thèse de doctorat qui devait porter sur l'histoire ${ }^{18}$, il introduit son analyse dans plusieurs de ces textes. L'historicisme scientifique a une allure positiviste que Péguy déprécie, c'est parce qu'il renvoie aux événements passés et archivés qui séloignent du réel, de la vraie source et de la vie. L'historiographie de Renan et de Taine frôle l'illusion et la prétention d'un « savoir momifié » (Bédarida 2002 : 104-106). Si Péguy condamne l'historicisme, il rend pourtant hommage à la mémoire qui renoue l'histoire à l'éternité (Bédarida 2002 : 106). Dans ce sens, l'histoire est à sauver à condition de lui prêter un regard plus profond et spirituel. La chronique des faits ne vaut donc pas une interprétation juste de l'histoire. Nous nous confrontons dans le poème Ève au « prévoyants » et aux « économes ", aux " experts » et "fins connaisseurs », " aux grands docteurs » et aux " bonnets d'âne » (Péguy 1957 : 1053-1054) qui tous contemplent enfin l’Enfant-rédempteur.

Dans l'essai Clio, Dialogue de l'histoire et de l'âme païenne, Péguy fait de l'histoire une vieille dame. Du coup, Ėve-aïeule vue dans le contexte de ses autres œuvres, est une vieillarde qui porte le joug non seulement du péché, mais aussi de l'histoire. Mais, de nouveau, dans la perspective de l'Incarnation que Péguy introduit, cette histoire qui s'ouvre au salut, se propose comme un héritage. Le poète insiste à travers ses répétitions : «Il allait hériter ..., Il allait hériter » (Péguy 1957 : 1070-1081).

Ève apporte une réflexion sur le péché originel. Cette inquiétude du poète face au péché ne se concentre pas et ne s'enferme pas seulement en la figure d'Êve. Ève, comme d'autres personnages des poèmes de Péguy, est de nature symbolique. Cette réflexion sur la " Mère des vivants » nous renvoie donc ailleurs. Elle éveille d'autres questions. Dans une vision plus globale, Ève représente donc la parabole de la chute et du péché de l'Homme. Elle incarne ainsi la condition humaine (Solignac 2015 : 7) ou, selon la formule de Camille Riquier, l'Humanité. « Ève, c'est l'humanité déchue qui parcourt les étapes d'une longue histoire qui va de la création jusqu’à la fin des temps, en traversant les climats du paradis, de la chute, de l'exil, de la crèche et du calvaire, avant de remonter la pente du temps et d'œuvrer au salut. » (Riquier 2015 : 183-205). Péguy explique lui-même cette voie du pécheur qui est humaine et qui est la sienne : "C'est le pécheur, c'est vous, c'est nous. C'est l'homme. Et essentiellement c'est le poète» (Péguy 1992 : 1220). Dans ce sens, Péguy déplore l'humanité, sa condition et son incapacité d'agir.

18 Cette idée du doctorat s’est substituée à un livre intitulé : «De la situation faite à l’histoire dans la philosophie générale du monde moderne" (1901-1909). 
Les autres n’ont connu que la basse maison.

Mais vous avez connu d’entrer dans cette tombe. (Péguy 1957 : 1005).

Le péché revient constamment dans la terminologie de Péguy. C’est l'habitude qui devient le péché, et c'est Ève qui était la " première assujettie à la loi d'habitude » (Péguy 1957 : 1004).

Il désigne souvent un désengagement de la pensée, une sorte d'inertie non seulement de l'individu, mais aussi celles de l'histoire et de l'humanité. Ce qui inquiète donc Péguy, ce avec quoi il n’arrive pas à être d'accord et ce dont il laisse les traces dans La note conjointe à Descartes, c'est la fermeture du monde moderne à la grâce de Dieu. C'est la modernité qui le trouble. Le péché fait donc tomber l'homme dans la modernité. Or, l'argent est un mauvais signe de cette modernité tournée vers le progrès et non pas vers la durée bergsonienne.

Le fait d'inscrire Ėve au sein de la modernité est, selon Riquier, un acte courageux. Le poème évoque le personnage biblique, la première femme du livre de la Genèse. La première femme « n’a plus connu » le bonheur idyllique de la vie sur terre. Péguy propage le sentiment d'une perte. Ève est chassée du paradis. Elle a perdu la félicité initiale. La condition d’Ève est irréparable selon Jean-Louis Chrétien (Chrétien 2015 : 85-110). Nous pensons tout de même que l'auteur du Porche du mystère de la deuxième vertu laisse de l'espérance qui est une vertu théologale et qui réfère ce qui est humain à ce qui est céleste.

\section{Conclusion}

La poésie de Charles Péguy réclame, dans la plasticité de la langue poétique, un souci de la personne humaine et de sa valeur. Par le procédé du retour à la figure d'Ėve, le poète sauve la mère des vivants, et, par la suite, la vie et la dignité humaines.

Dans ses œuvres, le poète ne se sert pourtant pas explicitement du terme "dignité humaine ", il défend et contemple une innocence première, originelle, qui pourrait être associée à cette dignité. Cette innocence est intrinsèque à l'Ėve du paradis. Péguy se penche charitablement sur la malheureuse condition d'Ėve biblique après la chute. La présence de Jésus à la fin des temps est comme la promesse d'une innocence reconquise et d'une heureuse éternité. La morale de Péguy se réfère bien aux valeurs chrétiennes qui dépassent, conformément à Bergson, l'ordre de la pensée rationnelle. Pourtant, Péguy propose de l'élever dans l'ordre de la grâce. Péguy établit le lien entre la personne et sa spiritualité. Dans cette optique, l'Homme peut espérer un avenir qui transgresse la temporalité.

Les bribes du bergsonisme et du personnalisme de Péguy se trouvent dans plusieurs de ses poèmes. Il mettra en valeur L'Espérance dans Le Porche du mystère de la deuxième vertu. Jeanne d'Arc, son héroïne privilégiée dans son innocence et sa faiblesse, reste toujours une promesse pour le monde. Péguy défend la fragilité, la féminité, la pauvreté ; il est ainsi conforme aux valeurs qui protègent l'Homme et qui s'accordent à la morale personnaliste qui sauvegarde la singularité humaine, «son véritable lieu est le cœur vivant des personnes " (Mounier 1965 : 88).

La pensée française à la charnière du XIX et du XX ${ }^{\mathrm{e}}$ siècle, aussi riche et variée soit-elle, contribue à la «valeur » de l'homme en accentuant sa singularité. La mise en lumière de l'aspect corporel et spirituel de l'humain, la valorisation de sa liberté, l'attention portée à sa fragilité et vulnérabilité 
renouent avec la sensibilité chrétienne. Fidèle à la philosophie bergsonienne, Péguy séduit par son idée de la durée pure, la transgresse vers le christianisme et les vertus théologales. Sa pensée va au-delà d'une morale fondée uniquement sur les valeurs sociales et terrestres. Elle se réfère aux valeurs transcendantes.

Ces visions auxquelles Charles Péguy se confronte, demeurent toujours comme un fil conducteur pour ses contemporains. Le bergsonisme et le personnalisme se heurtent, s'entrechoquent, se confrontent et rendent féconde la terre de la réflexion française. Ces grands philosophes français que sont Bergson et Mounier ont façonné une certaine forme de la pensée française du XX $\mathrm{X}^{\mathrm{e}}$ siècle. C'est une vision minoritaire mais qui garde son importance et son actualité.

\section{Références bibliographiques}

Azouvi, F. (2018). Bergson, Henri. In S. Malka (dir.), Dictionnaire Charles Péguy (pp. 43-48). Paris : Édition Albin Michel.

Bédarida, F. (2002). Histoire et mémoire chez Péguy, Presse de Sciences Po, «Vingtième Siècle. Revue d’histoire », 73, 101-110.

Bergson, H. (2012). Les deux sources de la morale et de la religion. Paris : Flammarion. - (1934). La Pensée et le mouvant. Paris : Librairie Félix Alcan.

Chantre, B. (2000). Péguy, l'essentiel médiateur. Le Magazine littéraire, 386, https://www.nouveau-magazine-litteraire.com/péguy-lessentiel-médiateur.

Chrétien, J.-L. (2015). La perte dans la pensée de Charles Péguy. In B. Chantre, C. Riquier, F. Worms (dir.), Pensée de Péguy (pp. 85-100). Paris-Perpignon : Desclée de Brouwer.

Cioran, E. (1964). La chute dans le temps. Paris : Gallimard.

Cottier, G. (2007). Le philosophe et la foi, Revue des sciences religieuses, 81, 307-321.

Delsol, Ch. (1914). Les Pierres d'angles. À quoi tenons-nous ? Paris : Cerf.

Dervin, F. (2010). Bergson, précurseur des mobilités académiques contemporaines ? Les Cahiers de Framespa. http://journals.openedition.org/framespa/589.

Gadacz, T. (2009). Historia filozofii wieku. Nurty. Filozofia życia, pragmatyzm, filozofia ducha. Kraków : Wydawnictwo Znak.

Granat, W. (1985). Personalizm chrześcijański. Teologia osoby ludzkiej. Poznań : Księgarnia św. Wojciecha.

Guéna, S. (2016). Introduction. In J. Maritain, E. Mounier (Eds.), Correspondance (pp. 9-24). Paris : Desclée de Brouer.

Hussherr, C. (2007). De l'Ėve biblique à l'Ève de Péguy. L’Amitié Charles Péguy. Acte du colloque sur Ève, $117-118,13-28$.

Kisukidi, N. Y. (2015). Familles spirituelles : Mounier - Péguy - Bergson. In E. Mounier, La Pensée de Charles Péguy (pp. 11-16). Paris : Édition du Félin.

Latour, B. (2015). Pourquoi Péguy se répète-t-il ? Péguy est-il illisible. In Camille Riquier (dir.), Charles Péguy. Les cahiers du Cerf (pp. 313-339). Paris : Cerf.

Le Gay, D. (2001). Les enjeux théologiques de deux fidélités chrétiennes. L’Amitié Charles Péguy. Péguy et Maritain. Une amitié à l'épreuve, 95, 13-28.

Lettre de Charles Péguy à Henri Bergson du 2 mars 1914 (1970). L’Amitiés Charles Péguy, 155, 48. 
Lettre de Henri Bergson à Daniel Halévy du 25 janvier 1939 (1970). L’Amitié Charles Péguy, 155, 56-57. Maritain, J. (1925). Trois réformateurs : Luther, Descartes et Rousseau. Paris : Plon.

Maritain, J.; Mounier, E. (2016). Correspondance. Paris : Desclée de Brouer.

Maritain, R. (2000). Les Grandes Amitiés. Souvenirs. Les Plans-sur-Bex : Éditions Paroles et Silence.

Mounier, E. (2015). La Pensée de Charles Péguy, Paris : Édition du Félin.

- (1965). Le personnalisme, Que sais-je. Paris : PUF.

Péguy, Ch. (1987). Euvres en proses complètes. t. I. Paris : Gallimard, NRF.

- (1992). Euvres en proses complètes. t. III. Paris : Gallimard, NRF. . (1957). Euvres poétiques complètes. Paris : Gallimard, NRF.

Pereira, K. M. K. (2005). Charles Péguy i Biblia. Lektura symboliczna życia i twórczości Péguy’ego. Warszawa : Wydawnictwo Neriton

Prelorentzos, I. (2007). Questions concernant la morale de Bergson, Philonsorbonne, 1, 103-129.

Riquier, C. (2007). Bergson et le problème de la personnalité : la personne dans tous ses états. Les Études philosophiques, 81, 193-214.

—. (2015). Ėve dans « Ève» - Lecture de Charles Péguy. In L. Solignac (dir.), La figure d'Adam (pp. 183-205). Paris : Hermann.

Rolland, R. (2015). Péguy. Paris : Éditions La Découverte.

Sołonko, N. (2019). Filozofia Henri’ego Bergsona w poezji Charlesa Péguy’ego. In A. Skala (dir.) Filozoficzne aspekty literatury. Studia i szkice (pp. 90-101). Lublin : Wydawnictwo Naukowe Tygiel.

Sertillages, A. D. (1941). Henri Bergson et le catholicisme. Paris : Flammarion.

Solignac, L. (2015). Avant-propos. Pour l'amour d'Adam. In L. Solignac (dir.), La figure d’Adam (pp. 5-13). Paris : Hermann.

Sueur, J.-P. (2007). Ėve : les vertiges de l'écriture. L’Amitié Charles Péguy. Acte du colloque sur Ève, 117-118, 85-106. 